\title{
Multi Drug Resistant Tuberculosis in Bhutan: A Look into The Line Probe Assay Results
}

\author{
Sonam Pelden ${ }^{1 *}$, Lila Maya Adhikari ${ }^{1}$, Kinley Gyem ${ }^{1}$, Pavitra Bhujel ${ }^{1}$, Tshering Dorji ${ }^{1}$, Ugyen \\ Dorji $^{1}$, Lekey Khandu ${ }^{2}$ and Jit Bdr Darnal ${ }^{1}$ \\ ${ }^{1}$ Royal Center for Disease Control, Bhutan
}

${ }^{2}$ National HIV/TB program, ministry of Health, Bhutan

*Corresponding author: Sonam Pelden, Royal Center for Disease Control, Chulabhorn College of Medicine, Thammasat University, Bhutan.

To Cite This Article: Sonam Pelden, Lila Maya Adhikari, Kinley Gyem, Pavitra Bhujel, Tshering Dorji, Ugyen Dorji, Lekey Khandu, Jit Bdr Darnal. Multi Drug Resistant Tuberculosis in Bhutan: A Look into The Line Probe Assay Results. Am J Biomed Sci \& Res. 2021 - 12(5). AJBSR. MS.ID.001792. DOI: 10.34297/AJBSR.2021.12.001792.

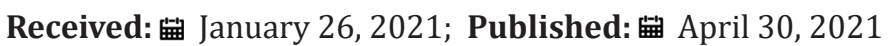

\begin{abstract}
Background: Bhutan plans to end TB by 2030 in line with the WHO End TB by 2030 goal. In 2016 National tuberculosis reference laboratory (NTRL) has performed Line probe assay (LPA) on 506 TB isolates, a huge jump from 326 cases in 2015.

Objectives: This review is aimed to understand the common mutations seen in the MDR isolates depicted by the line probe assay performed on the Mtb isolates from Bhutanese patients.
\end{abstract}

Methods: Line probe assay (LPA) results available from February 2014 to December 2016 at NTRL, has been retrospectively reviewed for drug resistance mutations. The data were of specimens from both from both pulmonary as well as extra pulmonary sites on which LPA has been performed.

Findings: Bhutan has seen an increase in the number of specimens referred in to NTRL for LPA and DST. Fifty-seven (14.4\%) MDR was seen in 2014, 16\% in 2017 and 10.7\% in 2016. Mono resistance to isoniazid was found more frequently compared to rifampicin mono-resistance by over 5 times. From our observation, MDRTB in Bhutanese isolates were mainly from the most frequently observed mutation worldwide, i.e at nt530533 (WT8), nt513-517 (WT3), and S531L (MUT3) of rpoB gene and at nt315 (WT1) and S315T1 (MUT1) of katG gene. Occasional involvement of inhA gene was found at -15/-16 (WT1), - 8 (WT2) and C15T (MUT1). More than 75\% of the MDR cases were due to dual mutations in the rpoB and katG genes (71.9\% in 2014, 86.5\% in 2015 and 88.9\% in 2016). Among all the 163 MDR isolates of the three consecutive years, the most frequent combination of mutation observed (127/163, 77.9\%) was the loss of rpoB WT8 (530-533) with rpoB MUT3 (S531L) and the loss of KatG WT1 (315) with KatG MUT1 (S315T1). The loss of rpoB WT8 (530-533) accounted for 93.9\% (153/163), closely followed by WT3 (513-517) with 82.2\% (134/163). WT7 (526-529) composed of 4.3\% (7/163 cases) of MDRTB. About 85\% (138/163) of rpoB MUT3 (S531L) was detected followed by 3 cases each of MUT2A and 2B. Ninety eight percent of MDR had WT bands missing and 93\% had katG MUT1 bands depicting mutations in S315T. katG MUT2, S315T2, was the most uncommon mutations with only a single case in 2014. No mutations or loss of ropB WT1, ropB WT2 and ropBWT6 have been detected in the MDR isolates, however in the RIF-MR no mutations were found from ropB WT1 through ropB WT6. Losses of ropB WT7 \& 8 were found to be the most common finding among the Bhutanese isolates. Mutations in katG commonly composed of the loss of WT with or without MUT1 and 2. The most common involvement in inhA gene was the loss of WT1 and WT2 bands with or without MUT1 band. However, no mutations in $k a t G$ and inhA were observed together at any location. Mutations in katG and inhA were however detected in MDR along with mutation in rpoB gene. Mutations in inhA, though less frequent in our MDR and INH Mono resistant isolates, it was reported to have a strong correlation with XDR TB in Western Cape and Eastern Cape Provinces, South Africa [1]. This is the first study where LPA depicted INH and RIF-drug-resistance-conferring mutations in MDR M. tuberculosis strains have been described.

Main Conclusion: LPA has facilitated diagnosis of MR/MDR TB and guide appropriate therapy. However, in resource limited country like Bhutan LPA has contributed immensely towards proper treatment for the patients.

Keywords: Tuberculosis; Multi drug resistance; Bhutan 


\section{Background}

An estimated 10.4 million new (incident) TB cases were reported worldwide in 2015 and 1.2 million (11\%) of all new TB cases were accounted among people living with HIV. The rate of decline in TB incidence remained at only 1.5\% from 2014 to 2015 [2] despite multinational efforts. Multi drug resistance tuberculosis (MDR-TB) is caused by Mycobacterium tuberculosis (Mtb) strains that are resistant to at least the first-line anti-tuberculous agents, rifampin (RIF) and isoniazid (INH). Additional resistance to at least one fluoroquinolone (FQ) and at least one of the three second line injectables; amikacin (AMK), capreomycin (CAP), and kanamycin (KAN) is referred to extremely drug resistance tuberculosis (XDRTB).

\section{Introduction}

Geographically, Bhutan is a tiny kingdom of 7,87,338 Bhutanese and an area of 38,394sq. kms [3] tucked away in the folds of the mighty Himalayas, at an altitude of $180 \mathrm{~m}-7,550$ meters above sea level. However, tuberculosis is an everyday battle like rest of the world. The mounting challenge through the years have been the increasing cases of MDR which has been among the population for decades now. National TB reference laboratory (NTRL) of the kingdom is equipped with various detection facilities from microscopy to genotypic line probe assay (LPA) for the diagnosis of both MDRTB.

INH is one of the cornerstones of anti-tuberculosis treatment, as it exhibits mycobactericidal activity by inhibiting mycolic acid biosynthesis. INH resistance commonly occurs due to mutations in the katG gene or the inhA regulatory regions. katG encodes catalase peroxidase, an enzyme that converts INH to its biologically active form. As mutations in katG, particularly at codon 315, confer high-level INH resistance, INH is ineffective for the treatment of Mycobacterium tuberculosis with this mutation profile. The inhA regulatory region encodes nicotin-amide adenine dinucleotidedependent enoyl-acyl carrier protein reductase, the primary target of active INH, as well as ethionamide (ETH) and prothionamide (PTH). inhA mutations cause low-level resistance to the drug, which means that high doses of INH may be effective against $M$. tuberculosis.

\section{Epidemiology and Emergence of MDR-TB in Bhutan}

Despite high prevalence of tuberculosis in neighboring Asian countries, Bhutan is among the low TB burden countries. Worldwide drug resistance has evolved due to poor understanding among those infected of the importance of adherence to treatment, quality of treatment as well as administrative and programmatic lapses. Moreover, other factors like social, demographical and geographical aspects at local or national levels, such as the development of the country play an enormous role in the epidemiology of tuberculosis in many countries alike [4]. Bhutan has a functional National Tuberculosis Control Program (NTCP) instituted in 1986 [5] and since then tuberculosis control activities have gradually been gearing up with awareness advocacies resulting in increased case detection. Tuberculosis in pediatrics, which is normally missed by many surveillances have however been well recognized in the country with excellent treatment outcome [6]. Furthermore, the rising HIV cases in the country, is a wakeup call for vigilance and renewed efforts to combat HIV-TB co-morbidities in the community. WHO reports 1.2 million (11\%) of all new TB cases in people living with HIV in 2015 [2]. However, there is no published data presenting the genetic studies of the clinical MTB isolates. With the institution of Line Probe Assay (LPA) in 2014, MDR-TB has been detected routinely helping the clinicians customize therapy for the $\mathrm{Tb}$ patients.

This review aims to present the mutations patterns occurring in the genes conferring mono-resistance to either INH or RIF (RR $\mathrm{DR}$ ); or to both; MDR, from the band patterns deciphered from the hybridization strips of the LPA from the past 3 years data from the Bhutanese population. The findings showed that mutations in the $r p o B$, katG, and inhA genes are similar to those reported from other parts of the world. Since no proper phenotypic and genotypic study is available in other parts of Bhutan, we cannot rule out the possibility of the existence of similar MDR or pre-XDR/XDR strains within the country. Due to inadequate monitoring and a lack of proper treatment regimens, MDR-TB and XDR-TB remain major threats to the Bhutanese population.

\section{Materials and Methods}

Line probe assay (LPA) results available from February 2014 to December 2016 at NTRL, has been retrospectively assessed for drug resistance. The data were of specimens of both from both pulmonary as well as extra pulmonary sites.

\section{Mycobacterium tuberculosis isolates}

Sputum or extra pulmonary specimens which were Acid fast positive by Zeihl Neelson staining are referred to NTRL for DST from the district hospitals and the Basic health units across the country. For this study a total of 293 isolates with valid LPA test results were used for further analysis of their resistance patterns after omitting 1021isolates which were found to be sensitive to INH and RIF or resulted in invalid tests on LPA.

\section{Culture and antibiotic susceptibility testing}

All smear positive specimens received in the laboratory within one week in cold chain were subjected to N-acetyl-Lcysteine-Sodium hydroxide (NALC-NaOH) method of digestion and decontamination. The specimen is then inoculated in Lowenstein Jensen (LJ) media and Mycobacteria Growth Indicator Tube (MGIT 960) automated culture methods. No solid media DST is 
performed at NTRL due to technical reasons. MGIT 960 Liquid media incorporated with $1.0 \mu \mathrm{g} / \mathrm{mL}$ of rifampicin and $0.1 \mu \mathrm{g} / \mathrm{mL}$ of isoniazid. In 2 weeks of incubation, the system indicated if the particular isolate was sensitive or resistant. Some specimens with $1+$ and above on Microscopic grading were directly subjected to LPA.

\section{DNA extraction and amplification}

Culture isolates from solid or liquid media or clinical specimens directly after decontamination are used for DNA extraction using GenoLyse ${ }^{\circledR}$ kit using manufacturer's instructions and amplified using Geno Type MDRTBplus, version 2 from Hain Lifesciences, Germany. LPAs are rapid tests used for the rapid detection of mutations in genes associated with drug resistance. This LPA is based on PCR and reverse hybridization methods that identifies M. tuberculosis complex and simultaneously detect mutations in $r p o B, K a t G$, and inhA genes in direct patient sputum or other specimen which confer resistance to RIF and INH. All the reagents for amplification including primers and polymerase are premixed for use. Test is validated using internal controls, Conjugate and Amplification Control. After reverse hybridization, the developed strips were aligned with the standard comparator provided with the test kit.

\section{Interpretation of results}

Each strip consists of 27 reaction zones (bands), including 6 controls (conjugate, amplification, $M$. tb complex, rpoB, katG, and inhA controls), 8 rроB wild-type [WT1-WT8 (506-509, 510-513, 513-517, 516-519, 518-522, 521-525, 526-529 and 530-533)] and 4 mutants [MUT1, 2A, 2B and 3 (D516V, H526Y, H526D and
S531L)], 1 katG WT (315) and 2 mutants [MUT1 and 2 (S315T1 and S315T2)] and 2 inhA WT [WT1 and 2 (-15/-16 and -8)] and 4 mutants [MUT1, 2, 3A and 3B (C15T, A16G, T8C and T8A)]. In general, for the 3 loci, a pattern comprising only WT bands was interpreted as sensitive. Resistance was interpreted as:

i) absence of $1 /$ more WT bands

ii) presence of mutant bands with or

iii) without the simultaneous absence of the complementary WT. The simultaneous presence of WT and corresponding mutant bands was referred to as a mixed pattern. The test detects monoresistance to isoniazid and rifampicin.

\section{Results}

The data includes reading from February 2014 as soon as the GenoType MDRTBplus, version 2 has been instituted in 2014 till that of December 2016. The number of specimens subjected to genotyping test each consecutive year was, 437, 341 and 535 in 2014, 2015 and 2016 respectively. However, several test have shown invalid LPA readings either due to missing control bands or due to the lack of amplification; $42 / 437$ (9.6\%) in 2014, 17/341 (4.9\%) in 2015 and 29/535 (5.42\%) in 2016 with either no control bands or missing bands altogether and have been excluded from the data analysis (Table 1). Data from solid culture DST was not available to complement the LPA findings. Figure 1 show the frequency of MDR, INH-monoresistance (INH-MR) and RIF-monoresistance (RIF-MR) isolated in the last 3 years. MDR has been constant along the years with an average of $\sim 14 \%$. Rifampicin monoresistance was found to be less frequent than INH monoresistance. In 2016, only a single case of RIF-MR was detected (Table 1 \& Figure 1).

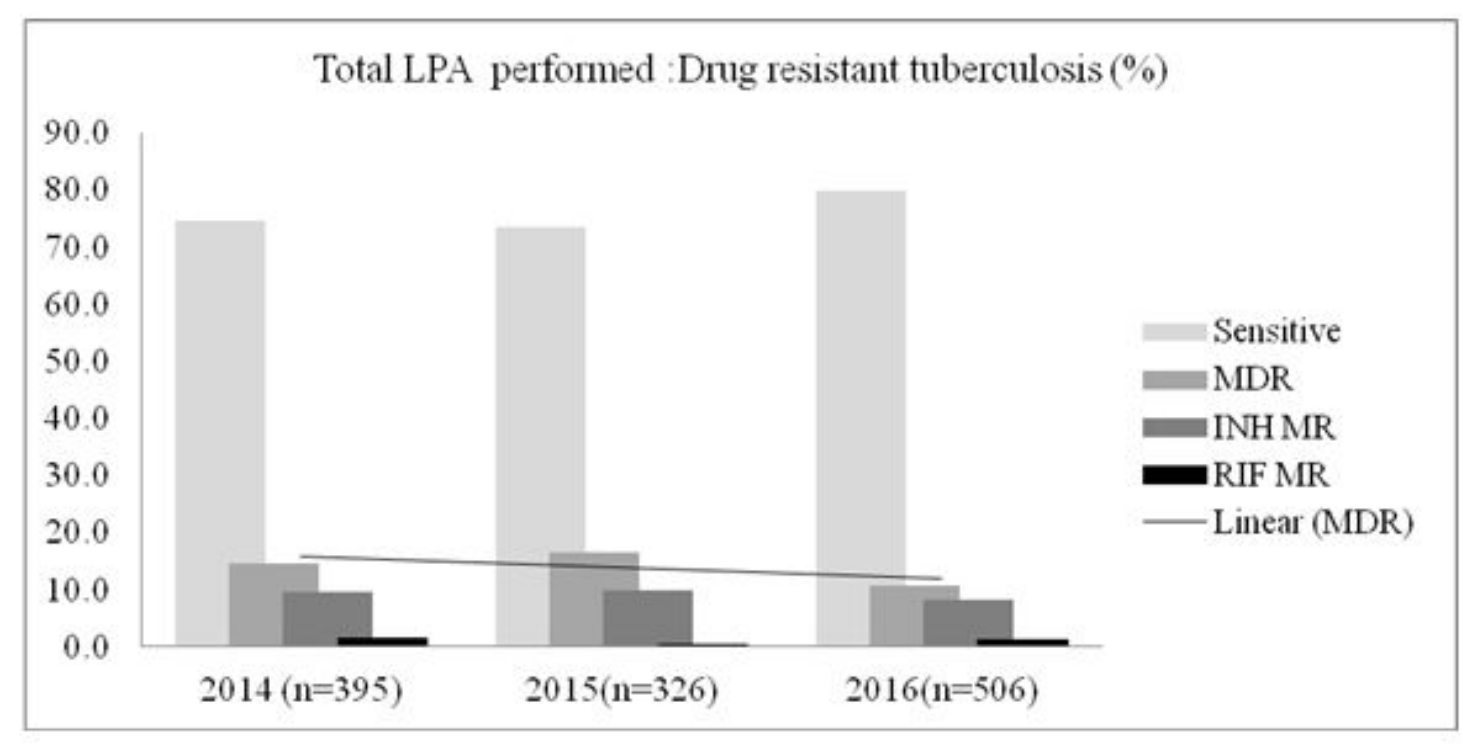

Figure 1: Drug resistant tuberculosis (\%) detected by line probe (hybridization) assay performed on the pulmonary and the extra pulmonary specimens following culture on solid and/liquid media or the tests performed on direct specimen as well. 
Table 1: Representation of the total number of specimens referred to NTRL for culture and DST from 2014 to 2016.

\begin{tabular}{|c|c|c|c|c|c|c|}
\hline & \multicolumn{2}{|c|}{2014} & \multicolumn{2}{|c|}{2015} & \multicolumn{2}{|c|}{2016} \\
\hline & No. of cases & $\%$ & No. of cases & $\%$ & No. of cases & $\%$ \\
\hline Sensitive & 294 & 74.4 & 239 & 73.3 & 404 & 79.8 \\
\hline MDR & 57 & 14.4 & 52 & 16 & 54 & 10.7 \\
\hline INH MR & 38 & 9.6 & 32 & 9.8 & 41 & 8.1 \\
\hline \multirow[t]{2}{*}{ RIF MR } & 6 & 1.5 & 1 & 0.3 & 7 & 1.4 \\
\hline & 395 & & 326 & & 506 & \\
\hline
\end{tabular}

\section{Detection of mutations in $r p o B, k a t G$ and $i n h A$ genes}

LPA has shown diverse combinations of wild type loss, mutation and mixed bands indicating mono resistances to INH and RIF or resistance to both the first line drugs. The most commonly observed band patterns in the MDR isolates were the mixed type [missing WT band (described here as WTL) with simultaneous detection of MUT bands] at both the $r p o B$ and the katG locus accounting for more than 70 percent of MDR-TB cases every consecutive year (41/57, $71.9 \%$ in $2014,45 / 52,86.5 \%$ in 2015 and $48 / 54,88.89 \%$ in 2016 . In 2014 the second commonest combination was rpoB WTL + katG (WTL+ MUT) $(11 / 57,19.3 \%)$. Mutations in inhA, though not very commonly detected in our isolates, have been detected in our MDR and INH-MR isolates. Three cases in 2014, 4 in 2015 and 3 in 2016 have shown mutation as well as loss of wild type bands in all the three genes [rpoB (WTL+MUT), katG (WTL+MUT), inhA WTL] and
[rpoB (WTL+MUT), katG (WTL+MUT), inhA MUT]. Furthermore in 2015 and 2016, a single case each of [(rpoB (WTL+MUT), inhA MUT)] and [rpoB (WTL+MUT), inhA (WTL+MUT)] with no mutations detected at the katG locus (Table 2). Majority of the INH resistance was observed to be contributed by both, loss of WT as well as due to mutations in the KatG gene. Loss of WT in katG alone was the second most common factor contributing ti INH MR in 2014 (15/38, not common with only $2 / 32$ (6.3\%) such cases detected in 2015. Mutations in inhA was occasional, furthermore not a single case of INH-MR was contributed by combined mutations in katG and inh $A$ together. From the entire three years data, it is not a single isolate had mutations in katG and inhA. However, one case in 2016 had mutations in $k a t G$ and $i n h A$ that has appeared together with that of mutations in rpoB (Table 2,3).

Table 2: Mutations in the rpoB, KatG and inhA genes. Diverse combinations of mutations conferring MDR to the isolates, for three consecutive years.

\begin{tabular}{|c|c|c|c|c|c|c|}
\hline & \multicolumn{2}{|c|}{2014} & \multicolumn{2}{|c|}{2015} & \multicolumn{2}{|c|}{2016} \\
\hline & No. of cases & $\%$ & No. of cases & $\%$ & No. of cases & $\%$ \\
\hline rpoB (WTL+MUT) + katG (WTL+MUT) & 41 & 71.9 & 45 & 86.5 & 48 & 88.9 \\
\hline rpoB MUT+ katG (WTL+ MUT) & 1 & 1.8 & 0 & 0 & 1 & 1.9 \\
\hline rpoB (WTL+ MUT) + kat G (WTL+MUT) + inhA (WTL+MUT) & 0 & 0 & 0 & 0 & 1 & 1.9 \\
\hline rpoB (WTL+MUT) + KatG MUT & 0 & 0 & 0 & 0 & 1 & 1.9 \\
\hline rpoB WTL+ katG (WTL+MUT) & 11 & 19.3 & 1 & 1.9 & 2 & 3.7 \\
\hline rpoB (WTL+MUT) + inhA (WTL+ MUT) & 1 & 1.8 & 1 & 1.9 & 0 & 0 \\
\hline rpoB (WTL+MUT) + KatG WTL & 2 & 3.5 & 2 & 3.8 & 1 & 1.9 \\
\hline \multirow[t]{2}{*}{ rpoB WTL+katG WTL } & 1 & 1.8 & 3 & 5.8 & 0 & 0 \\
\hline & 57 & & 52 & & 54 & \\
\hline
\end{tabular}

Table 3: Mutations in katG and inhA giving rise to INH monoresistance.

\begin{tabular}{|c|c|c|c|c|c|c|}
\hline & \multicolumn{2}{|c|}{2014} & \multicolumn{2}{|c|}{2015} & \multicolumn{2}{|c|}{2016} \\
\hline & No. of cases & $\%$ & No. of cases & $\%$ & No. of cases & $\%$ \\
\hline katG WTL + katG MUT & 14 & 36.8 & 28 & 87.5 & 35 & 85.4 \\
\hline katG WTL & 15 & 39.5 & 0 & 0 & 1 & 2.4 \\
\hline katG MUT & 7 & 18.4 & 0 & 0 & 3 & 7.3 \\
\hline inhA WT + inhA MUT & 0 & 0 & 3 & 9.4 & 0 & 0 \\
\hline inhA MUT & 1 & 2.6 & 0 & 0 & 0 & 0 \\
\hline
\end{tabular}




\begin{tabular}{|c|c|c|c|c|c|c|}
\hline inhA WTL & 1 & 2.6 & 1 & 3.1 & 2 & 4.9 \\
\hline Total INH MR & 38 & & 32 & & 41 & \\
\hline
\end{tabular}

Table 4: Rifampicin monoresistance.

\begin{tabular}{|c|c|c|c|c|c|c|}
\hline & \multicolumn{2}{|c|}{2014} & \multicolumn{2}{|c|}{2015} & \multicolumn{2}{|c|}{2016} \\
\hline & No. of cases & $\%$ & No. of cases & $\%$ & No. of cases & $\%$ \\
\hline rpoB WTL + rpoB MUT & 2 & 33.3 & 1 & 100 & 3 & 42.9 \\
\hline rpoB WTL & 4 & 66.7 & 0 & 0 & 4 & 57.1 \\
\hline rpoBMUT & 0 & 0 & 0 & 0 & 0 & 0 \\
\hline Total Rif-MR & 6 & & 1 & & 7 & \\
\hline
\end{tabular}

We observed that monoresistance to RIF was not common compared to monoresistance to INH (Table 1), however mutations in the $r р о B$ are seen commonly with mutations in katG (Table 4). Similar to our observation Deepa P et. al. 2005, reported an association between RIF resistant strains and the resistance to INH in majority of the cases therefore the feasibility of using RIF resistance as a surrogate marker of MDR [7]. In 2015 only one case of RIF-MR was detected and 7 cases in 2016. Unlike RIF, monoresistance to INH was a common occurrence (38 cases in 2014, 32 cases in 2015 and 41 cases in 2016).

Among all the 163 MDR isolates of the three consecutive years, the most frequent combination of mutation observed $(127 / 163$, $77.9 \%$ ) was the loss of rpoB WT8 (530-533) with rpoB MUT3 (S531L) and the loss of katG WT1 (315) with katG MUT1 (S315T1). The loss of rров WT8 (530-533) accounted for 93.9\% (153/163), closely followed by WT3 (513-517) with 82.2\% (134/163). WT7 (526-529) composed of 4.3\% (7/163 cases) of MDRTB. About $85 \%$ (138/163) of rpoB MUT3 (S531L) was detected followed by 3 cases each of MUT2A and 2B. Ninety eight percent of MDR had WT bands missing and 93\% had katG MUT1 bands depicting mutations in S315T. katG MUT2, S315T2, was the most uncommon mutations with only a single case in 2014 (Table 5). No mutations or loss of ropB WT1, ropB WT2 and ropBWT6 have been detected in the MDR isolates, however in the RIF-MR no mutations were found from ropB WT1 through ropB WT6 (Table 6). Losses of WT7 \& 8 of $\operatorname{rop} B$ gene were found to be the most common finding among the Bhutanese isolates. Mutations in katG commonly composed of the loss of WT with or without MUT1 and 2 (Table 6). The most common involvement in inhA gene was the loss of WT1 and WT2 bands with or without MUT1 band.

Table 5: Band patterns observed in $\mathrm{rpoB}$, katG and inhA gene $(n=163)$ of MDR isolates. Since the mutations are found in various combinations, the total need not work out to be equal to 163 isolates.

\begin{tabular}{|c|c|c|}
\hline MDR isolates $(n=163)$ & Freq. of Mutations & $\%$ \\
\hline \multicolumn{3}{|c|}{ katG } \\
\hline WTL & 160 & 98.16 \\
\hline MUT1 & 152 & 93.25 \\
\hline MUT2 & 1 & 0.61 \\
\hline \multicolumn{3}{|c|}{ ropB } \\
\hline WT3 & 134 & 82.2 \\
\hline WT4 & 3 & 1.8 \\
\hline WT5 & 3 & 1.8 \\
\hline WT7 & 7 & 4.3 \\
\hline WT8 & 153 & 93.9 \\
\hline MUT2A & 3 & 1.8 \\
\hline MUT2B & 3 & 1.8 \\
\hline MUT3 & 138 & 84.7 \\
\hline \multicolumn{3}{|c|}{ inhA } \\
\hline WT1 & 3 & 1.84 \\
\hline
\end{tabular}




\begin{tabular}{|c|c|c|}
\hline WT2 & 2 & 1.23 \\
\hline MUT1 & 3 & 1.84 \\
\hline
\end{tabular}

Table 6: Overall band patterns of drug resistant Mycobacterium tuberculosis strains using line probe assay.

\begin{tabular}{|c|c|c|c|c|c|}
\hline Gene & Band & Region of mutation & RIF MR (n=14) & INH MR (n=111) & MDR $(n=163)$ \\
\hline \multicolumn{6}{|c|}{ rpoB } \\
\hline & WT1 & $506-509$ & $14(100)$ & 111(100) & $163(100)$ \\
\hline & WT2 & $510-513$ & $14(100)$ & $111(100)$ & $163(100)$ \\
\hline & WT3 & 513-517 & $14(100)$ & $111(100)$ & $134(82.2)$ \\
\hline & WT4 & 516-519 & $14(100)$ & $111(100)$ & $3(1.8)$ \\
\hline & WT5 & $518-522$ & $14(100)$ & $111(100)$ & $3(1.8)$ \\
\hline & WT6 & $521-525$ & $14(100)$ & $111(100)$ & $163(100)$ \\
\hline & WT7 & $526-529$ & $6(42.9)$ & $111(100)$ & $7(4.3)$ \\
\hline & WT8 & $530-533$ & $9(64.3)$ & $111(100)$ & $153(93.9)$ \\
\hline & MUT1 & D516V & $0(0)$ & $0(0)$ & $163(100)$ \\
\hline & MUT2A & H526Y & $2(14.3)$ & $0(0)$ & $3(1.8)$ \\
\hline & MUT2B & H526D & $4(28.6)$ & $0(0)$ & $3(1.8)$ \\
\hline & MUT3 & S531L & $0(0)$ & $0(0)$ & 138(84.7) \\
\hline \multicolumn{6}{|c|}{ katG } \\
\hline & WT & 315 & $14(100)$ & $87(78.4)$ & $61(37.4)$ \\
\hline & MUT1 & S315T1 & $0(0)$ & $93(83.8)$ & $52(31.9)$ \\
\hline & MUT2 & S315T2 & $0(0)$ & $0(0)$ & $1(0.6)$ \\
\hline \multicolumn{6}{|c|}{ inhA } \\
\hline & WT1 & 0.9375 & $14(100)$ & $7(6.3)$ & $3(1.8)$ \\
\hline & WT2 & -8 & $14(100)$ & $2(1.8)$ & $2(1.2)$ \\
\hline & MUT1 & $\mathrm{C} 15 \mathrm{~T}$ & $0(0)$ & $4(3.6)$ & $3(1.8)$ \\
\hline & MUT2 & A16G & $0(0)$ & $0(0)$ & $0(0)$ \\
\hline & MUT3A & $\mathrm{T} 8 \mathrm{C}$ & $0(0)$ & $0(0)$ & $0(0)$ \\
\hline & MUT3B & T8A & $0(0)$ & $0(0)$ & $0(0)$ \\
\hline
\end{tabular}

Percentage values are shown in parentheses.

RIF: Rifampicin; INH: Isoniazid; MDR: Multidrug Resistant-Pulmonary and Extra Pulmonary TB

\section{Discussion}

Tuberculosis is a global health issue of huge concern. Curtailing MDRTB transmission is the only best solution to controlling tuberculosis epidemic. Transmission of TB bacilli and most dangerous in the recent years, MDR/XDR mycobacteria can only be controlled by correctly diagnosing and treating the patients. Bhutan has seen an increase in the specimens referred in to NTRL for DST. LPA has been a boon, facilitating early diagnosis of MR/ MDR TB and guide appropriate therapy. From our observation, MDRTB in Bhutanese isolates mainly harbored the most frequently observed mutation at nt530-533 (WT8), nt513-517 (WT3), and S531L (MUT3) of rpoB gene and at nt315(WT1) and S315T1 (MUT1) of katG gene. Resistance to INH or mutations in the katG gene (particularly S315T) has been reported to have evolved prior to the resistance to RIF [8]. Occasional involvement of inhA gene was found -15/-16 (WT1), -8 (WT2) and C15T (MUT1). However, no mutations in katG and inhA were observed together at any location. Mutations in $k a t G$ and inhA were however detected in MDR with mutation in $r p o B$ gene. Coincidentally M. Muthaiah et al. also reported, only two strains had mutations in both the katG and inhA genes [9]. Since isoniazid resistance is largely believed to be the first acquired resistance of all anti-tubercle drugs [10], early diagnosis and management of mutations in $k a t G$ and $i n h A$ is indispensible to curb the progression of resistances to other drugs. This theory overrides the importance of Gene Xpert machines for RIF resistance screening, at which point RIF resistance is already gained and MDR already set in. Availability of gene sequencing studies would reveal resistance patterns and help phylogenetic analysis of our strains. Mutations in inhA, though less frequent in our MDR and INH MR 
isolates, it was reported to have a strong correlation with XDR TB in Western Cape and Eastern Cape Provinces, South Africa [1]. Many reported a low-level resistance associated with the mutations in the inhA which could be therapeutically overcome with high dose isoniazid [11,12]. India, the closest neighbor who is among the 30 countries of high TB burden countries in the world has reported 79,000 drug resistant Tb cases in 2015 among which 28,876 were MDR/RR-TB and 3048 were XDR-TB [13]. Free permeable border allows, intensive cross border trade and close interaction, with a high possibility of transmitting TB bacilli among the people. It would be interesting to delve into the epidemiological and genetic study to see associations between our strains thereby helping cross-border advocacy and refine control policies. However, it is also important to note that $M$. $t b$ strains exhibit geographical variation. Patra et al. observed different mutation patterns in RRDR of $r p o B$ studied at the same location depicting changes in mutation profiles [14]. Such rapid tests require designing of probes based on the knowledge of mutation profile in different geographical area, however the constantly changing resistance patterns may be a challenge in achieving a fool-proof test kit for a particular region. With no data on mutations $M$. tuberculosis strains in Bhutan, it is unsure if this rapid device had missed out on any isolates with unusual mutations beyond the scope of this device. Genotype MDRTBplus has a reported sensitivity of $95.29 \%$ and specificity of $95.16 \%$ for detecting MDRTB. The sensitivity and specificity for the detection of INH and RIF resistance was 89.29\% \& 95.95\% and $91.98 \%$ \& 95.79\% respectively [15]. Madhuri K et al., 2015 has reported a sensitivity of $98.1 \%$ and specificity of $97.8 \%$ for detection of rifampicin resistance, and $92.1 \%$ sensitivity and $97.9 \%$ specificity for the detection of isoniazid resistance [16]. Likewise, most of the literatures have reported sensitivities and specificities above $90 \%$ tested in different regions of the world [15]. However, Tolani et al., 2012, reported a sensitivity and spec of 83.3 and $100 \%$ for the detection of rifampicin resistance and $85.7 \%$ and $100 \%$ for the detection of isoniazid resistance [17].

This is the first study where LPA depicted INH and RIF-drugresistance-conferring mutations in MDR M. tuberculosis strains have been described. Now with the shifting of focus from diagnosis to the prediction of possible resistances that would be acquired by the isolates [10], curbing of tuberculosis seems promising. However, in resource limited country like Bhutan LPA has contributed immensely towards proper treatment for the patients. Bhutan is rapidly developing with increased human mobility.

\section{Limitations}

i. No phenotypic DST data available for comparison and Loss of valuable data in the invalid results.

ii. Therefore low, intermediate or high-level resistance cannot be determined. iii. LPA does not cover unidentified mutations in other genomic regions (like $a h p C$, kasA, furA).

iv. Data was available only for 3 years as LPA was only recently, therefore changes in the band patterns over many years cannot be compared.

\section{Conclusion}

Mutation at codon 315 of $k a t G$ gene is the major cause for isoniazid (INH) resistance M. tuberculosis. Errors in the Tuberculosis diagnosis are risky and expensive with prolonged anti-tuberculosis therapy. Although rapid molecular tests have advantageous attributes, it is always commendable to complement its results with the findings from the solid culture DST. For the same purpose most, laboratories have adapted to dual method of detecting drug resistance TB. The present study, although limited by the small sample size, is however concerning, and additional studies are needed to more accurately define the prevalence of such resistant strains in both pulmonary and extra-pulmonary materials among the population. Bhutan is yet to have enough information on the genetic background or the lineages of the commonly circulating strains of mycobacterium tuberculosis. With mounting volume of literatures and findings from every corner of the world, with differing methodologies and population background, it is almost impossible to adapt to one single finding. Even within a region, the resistance mutations and patterns are not constant. Therefore, further studies, using DNA sequencing, are needed to characterize these mutations in our own region for treatment and policy guidance.

\section{Acknowledgement}

None.

\section{Conflict of Interest}

No conflict of interest.

\section{References}

1. Müller BS, Hoek EM, Tait KGP, Trollip M, Bosman A, et al. (2011) inhA promoter mutations: a gateway to extensively drug-resistant tuberculosis in South Africa? Int J Tuberc Lung Dis 15(3): 344-351.

2. (2016) Organization WH. World Health Organization: Global actions and investments fall far short of those needed to end the global TB epidemic. Global Tuberculosis report.

3. (2017) National Statistics Bureau, Royal Government of Bhutan. Statistical yearbook of Bhutan 2016.

4. Rodriguez Morales DMC HaAJ (2013) Epidemiological Burden of Tuberculosis in Developing Countries. Intech: 24.

5. (2010) National Tuberculosis Control Programme, Department of Public Health, Ministry of Health, Royal Government of Bhutan. Guidelines for the management of tuberculosis. ( $5^{\text {th }}$ edn), NTCP, Thimphu, Bhutan.

6. Dendup T, Dorji T, Edgnton ME, Kumar AM, Wangchuk D, et al. (2013) Childhood tuberculosis in Bhutan: profile and treatment outcomes. Public health action 3(1): 11-14. 
7. Deepa KLTaHNM (2005) Detection and characterization of mutations in rifampicin resistant mycobacterium tuberculosis clinical isolates by DNA sequencing. Indian Journal of Tuberculosis 52: 5 .

8. Harris KA Jr, Mukundan U, Musser JM, Kreiswirth BN, Lalitha MK (2000) Genetic diversity and evidence for acquired antimicrobial resistance in Mycobacterium tuberculosis at a large hospital in South India. Int J Infect Dis 4(3): 140-147.

9. Muthaiah M, Shivekar SS, Cuppusamy Kapalamurthy VR, Alagappan C, Sakkaravarthy A, et al. (2017) Prevalence of mutations in genes associated with rifampicin and isoniazid resistance in Mycobacterium tuberculosis clinical isolates. J Clin Tuberc Other Mycobact Dis 8: 19-25.

10. Manson AL, Cohen KA, Abeel T, Desjardins CA, Armstrong DT, et al. (2017) Genomic analysis of globally diverse Mycobacterium tuberculosis strains provides insights into the emergence and spread of multidrug resistance. Nat Genet. 49(3): 395-402.

11. Jacobson KR, Theron D, Victor TC, Streicher EM, Warren RM, et al. (2011) Treatment Outcomes of Isoniazid-Resistant Tuberculosis Patients, Western Cape Province, South Africa. Clin Infect Dis 53(4): 369-372.

12. Niehaus AJ, Mlisana K, Gandhi NR, Mathema B, Brust JCM (2015) High Prevalence of inhA Promoter Mutations among Patients with Drug-
Resistant Tuberculosis in KwaZulu-Natal, South Africa. PLoS One 10(9): e0135003.

13. (2016) WHO World Health Organization; Country profiles for 30 high burden countries. Global Tuberculosis Report.

14. Patra SK JA, Sherwal BL, Khanna A (2010) Rapid detection of mutation in RRDR of rpoB gene for rifampicin resistance in MDR-pulmonary tuberculosis by DNA sequencing. Indian J Clin Biochem 25(3): 315-318.

15. Nikam C, Patel R, Sadani M, Ajbani K, Kazi M, et al. (2016) Redefining MTBDRplus test results: What do indeterminate results actually mean? Int J Tuberc Lung Dis 20(2): 154-159.

16. Madhuri K, Deshpande S, Dharmashale S, Bharadwaj R (2015) Utility of Line Probe Assay for the Early Detection of Multidrug-Resistant Pulmonary Tuberculosis. J Glob Infect Dis 7(2): 60-65.

17. Tolani MP, D Souza D T, Mistry NF (2012) Drug resistance mutations and heteroresistance detected using the GenoType MTBDRplus assay and their implication for treatment outcomes in patients from Mumbai, India. BMC Infect Dis 12: 9. 